\title{
The Impact of COPD on in-Hospital Outcomes in Patients with Takotsubo Cardiomyopathy
}

This article was published in the following Dove Press journal:

International Journal of Chronic Obstructive Pulmonary Disease

\author{
Pengyang $\mathrm{Li}^{1, *}$ \\ Xiaojia $\mathrm{Lu}^{2, *}$ \\ Catherine Teng $\mathbb{1}^{3}$ \\ Peng $\mathrm{Cai}^{4}$ \\ Mark Kranis ${ }^{5}$ \\ Qiying Dai ${ }^{5}$ \\ Bin Wang $\mathbb{D}^{2}$
}

'Department of Medicine, Saint Vincent Hospital, Worcester, MA 01608, USA;

${ }^{2}$ Department of Cardiology, The First Affiliated Hospital of Shantou University Medical College, Shantou, Guangdong,

5I504I, People's Republic of China;

${ }^{3}$ Department of Internal Medicine, Yale

New Haven Health-Greenwich Hospital,

Greenwich, CT 06830, USA;

${ }^{4}$ Department of Mathematical Sciences,

Worcester Polytechnic Institute,

Worcester, MA 01609, USA; ${ }^{5}$ Division of

Cardiology, Saint Vincent Hospital,

Worcester, MA 01608, USA

*These authors contributed equally to this work

\begin{abstract}
Purpose: Chronic obstructive pulmonary disease (COPD) is a known comorbidity of takotsubo cardiomyopathy (TCM), and COPD exacerbation is a potential triggering factor of TCM. The association between COPD and in-hospital outcomes and complications among TCM patients is not well established. We sought to assess the effect of COPD on hospitalized
\end{abstract} patients with a primary diagnosis of TCM.

Methods: We conducted a retrospective cohort study in patients with a primary diagnosis of TCM with or without COPD using the latest National Inpatient Sample from 2016-2017. We identified 3139 patients admitted with a primary diagnosis of TCM by the ICD-10-CM coding system; 684 of those patients also had a diagnosis of COPD. We performed propensity score matching in a 1:2 ratio $(n=678$ patients, matched COPD group; $n=1070$, matched non-COPD group) and compared in-hospital outcomes and complications between TCM patients with and without a COPD diagnosis.

Results: Before matching, the COPD group had worse outcomes compared with the nonCOPD group in inpatient death $(2.9 \%$ vs $1.3 \%, p=0.006)$, length of stay (LOS) $(4.02 \pm 2.99$ days vs $3.27 \pm 3.39$ days, $\mathrm{p}<0.001)$, hospitalization charges $(\$ 55,242.68 \pm 47,637.40$ vs $\$ 48,316.97 \pm 47,939.84, \mathrm{p}=0.001)$, and acute respiratory failure (ARF) $(22.5 \%$ vs $7.7 \%$, $\mathrm{p}<0.001$ ), respectively. After propensity score matching, the matched COPD group, compared with the matched non-COPD group, had a higher inpatient mortality rate $(2.9 \%$ vs $1.0 \%, \mathrm{p}=0.005)$, longer LOS ( $4.02 \pm 3.00$ days vs $3.40 \pm 3.54$ days, $\mathrm{p}<0.001)$, higher hospitalization charges $(\$ 55,409.23 \pm 47,809.13$ vs $\$ 46,469.60 \pm 42,209.10, \mathrm{p}<0.001)$, and a higher incidence of ARF $(22.6 \%$ vs $8.2 \%, \mathrm{p}<0.001)$ and cardiogenic shock $(5.6 \%$ vs $3.3 \%$, $\mathrm{p}=0.024)$, respectively.

Conclusion: Patients with COPD who are hospitalized for TCM have higher rates of inpatient mortality, ARF, cardiogenic shock, as well as a longer LOS, and higher charges of stay than those without COPD. Prospective studies are warranted to examine the effect of early intervention or treatment of COPD on short- and long-term outcomes of TCM.

Keywords: takotsubo cardiomyopathy, chronic obstructive pulmonary disease, in-hospital outcomes

\section{Introduction}

Takotsubo cardiomyopathy (TCM), first described in Japan in 1990, is characterized by regional wall motion abnormalities that extend beyond a single coronary artery distribution, and a lack of evidence of coronary artery occlusion. ${ }^{1}$ The term "takotsubo" in Japanese refers to an octopus trap, which resembles the typical finding of the left ventricle in systole on echocardiograms. ${ }^{2}$ TCM's clinical presentation, electrocardiographic findings, and biomarker profiles resemble acute myocardial infarction (AMI). ${ }^{1,3,4}$ Once thought to be a temporary, reversible
Department of Cardiology, The First Affiliated Hospital of Shantou University Medical College, 57 Changping Road,

Shantou, Guangdong 5I504I, People's Republic of China

Tel +86-75488905399

Fax +8675488259850

Email wangbin_pku@।26.com
International Journal of Chronic Obstructive Pulmonary Disease 2020:I5 2333-234I

2333

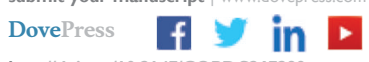

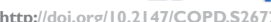


condition, TCM has been found to have a mortality rate similar to acute coronary syndrome. ${ }^{3}$ Approximately $1-2 \%$ of individuals who initially present with AMI patients are in fact sufferers of TCM. ${ }^{5}$ In addition, the TCM hospitalization rate has increased from 5.7 per 100,000 personyears in 2007 to 17.4 in $2012 .{ }^{6}$ Many pre-existing factors have been identified that affect the outcomes of TCM include age, ${ }^{7}$ sex,${ }^{8}$ race, ${ }^{9}$ conventional cardiovascular risk factors (such as obesity, ${ }^{10}$ and diabetes mellitus $(\mathrm{DM})^{11}$ ), psychiatric disorders (such as anxiety, depression ${ }^{12}$ ), and chronic kidney disease (CKD). ${ }^{13,14}$

Chronic obstructive pulmonary disease (COPD) is one of the leading causes of death worldwide. ${ }^{15,16}$ It is often seen in patients with TCM, with an even higher prevalence in patients with acute coronary syndrome. ${ }^{17-20}$ Many studies have found that TCM could be triggered by acute exacerbation of COPD, though the evidence of COPD affecting the outcomes of admitted TCM patients is lacking. ${ }^{21-23}$ In this study, we used the latest data available in the National Inpatient Sample (NIS) 2016 and 2017 the largest inpatient discharge database in the United States - to identify the association between COPD and the inpatient outcomes of TCM.

\section{Methods}

\section{Data Source}

The NIS database, compiled by the Agency for Healthcare Research and Quality (AHRQ), includes more than 7 million patients per year in the United States, and approximates a $20 \%$ stratified sample of all discharges from US community hospitals (excluding rehabilitation and long-term acute care hospitals). ${ }^{24}$ It contains information on patient demographics, administrative codes for primary diagnosis and secondary diagnoses, procedures, discharge status, disposition, hospital charges, and length of stay (LOS). It has been widely used in examining the utilization of hospital health services, practice variation, cost, and the impact of health policy interventions in the inpatient setting. ${ }^{25}$ As a de-identified publicly available database, Institutional Review Board (IRB) approval is not required in using NIS. Beginning with the data year 2016, the NIS includes a full calendar year of data with diagnosis codes reported using the International Classification of Diseases, Tenth Revision, Clinical Modification (ICD-10-CM) coding system. NIS 2016 and 2017 are the latest available NIS using the ICD-10-CM coding system.

\section{Study Population}

All patients admitted to a hospital from January 1, 2016 to December 31, 2017 with the primary diagnosis of TCM were selected in this study, using ICD-10-CM codes (Supplementary Table 1). Patients without discharge status were excluded. Eligible patients in this study were designated into two groups based on whether or not the patient carried a diagnosis of COPD. The process of patient selection in this study is shown in Figure 1.

\section{Study Variables}

Patients' demographic data were collected from NIS 2016 and 2017, including age, sex, race, geographic location, household income, primary payer, hospital type, region

National Inpatient Sample Database (2016-2017)

$(\mathrm{N}=14,294,784)$

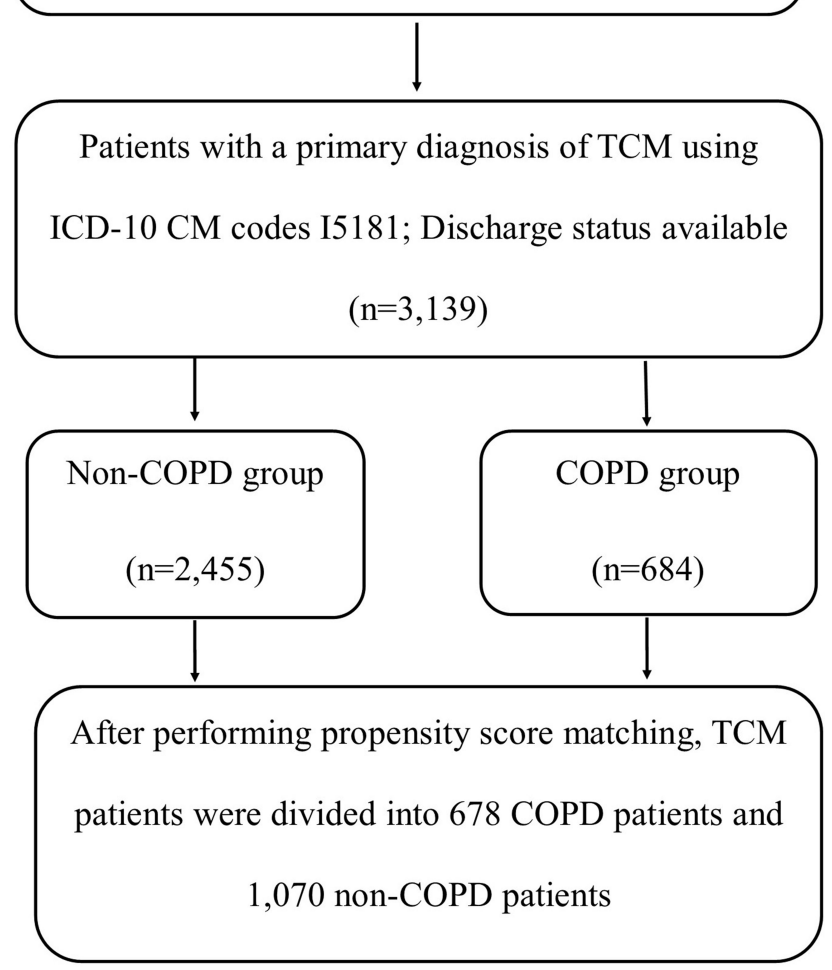

Figure I Flow chart of the selection process for the final patient sample used in this study.

Notes: We selected 3139 patients with a primary diagnosis of TCM from the 2016 and 2017 NIS database using the ICD-10-CM of 15I8I. All eligible patients were divided into two groups based on whether they had the diagnosis of COPD; 684 patients were in the COPD group and 2455 in the non-COPD group. After the propensity score matching in a target ratio of I:2, 678 COPD patients and 1070 non-COPD patients remained. We then compared inpatient outcomes between two groups.

Abbreviations: TCM, takotsubo cardiomyopathy; COPD, chronic obstructive pulmonary disease; ICD-I0-CM code, Tenth Revision, Clinical Modification Code. 
and bed size. Comorbidities collected include hypertension, DM, hyperlipidemia, obesity, smoking, obstructive sleep apnea (OSA), depression, anxiety disorders and CKD, by the ICD-10-CM codes from the discharge diagnosis. (Supplement Table 1)

\section{Outcomes}

The primary outcome of this study is inpatient mortality. The secondary outcomes were LOS, total charges of stay, and certain severe inpatient complications: cardiac arrest, cardiogenic shock, ventricular arrhythmia, acute kidney injury (AKI), and acute respiratory failure (ARF).

\section{Statistical Analyses}

Descriptive data are shown in percentages or mean \pm standard deviation (SD), and tested with the chi-square and $t$-test for categorical and continuous variables, respectively.

To reduce the bias in the unmatched data, we use propensity score matching analysis to match patients from the COPD group and the non-COPD group. Compared to a multivariable regression model, the propensity score matching model has the following advantages: 1) the number of confounders used in propensity score matching is not limited by the number of outcome events, 2) propensity score matching eliminates the linearity assumption between the propensity score and outcome, and 3 ) estimates the treatment effect by modelling covariates and treatment assignment. It mirrors a randomized experiment by separating the study design from the outcome analysis, which protects against actual or suspected bias from the researchers. ${ }^{26}$

To measure propensity scores, we built a multivariate logistic regression model and adjusted it for individual characteristics (age, sex, race, and mean household income), hospital-level characteristics (hospital type, region, and size) and the comorbidities mentioned above, using the nearest neighbor matching of both groups with a caliper match tolerance of 0.05 . A match ratio of $1: 2$ (COPD: non-COPD patients) was used based on initial findings, as there were 684 TCM patients with COPD and 2455 without. In other words, for each TCM patient with COPD, an effort was made to match the patient with two patients without COPD with similar demographic characteristics. After matching, 678 patients with COPD were identified, along with 1070 patients without COPD. Finally, we compared the in-hospital outcomes and complications between the COPD and non-COPD groups in both the unmatched and the propensity score-matched cohorts.

All statistical analysis was performed by the $\mathrm{R}$ statistics software (version 3.6.1, R Development Core Team). The matching process was conducted using the MatchIt package in $\mathrm{R}$ software. All tests were two-sided. The results were considered significant at $\mathrm{p}<0.05$.

Standardized mean difference is used to examine the balance of the covariate distribution between matched COPD group and non-COPD group. Standardized mean differences less than 0.1 were considered balanced. ${ }^{27}$

\section{Results}

\section{Baseline Characteristics}

3139 patients with a primary diagnosis of TCM from the 2016 and 2017 NIS database were identified. Among them, 684 carried the comorbidity of COPD while 2455 did not. Once matched in a 1:2 target ratio, there are 678 patients in COPD subgroup and 1070 in non-COPD subgroup.

In the unmatched cohorts, the majority of patients in both groups were female (89.2\% in COPD group, $90.8 \%$ in non-COPD group, $\mathrm{p}=0.306)$. Compared with the nonCOPD group, TCM patients with COPD tended to be older $(68.07 \pm 10.47$ vs. $66.28 \pm 13.21, \mathrm{p}=0.001)$, whiter (82.3\% vs. $78.7 \%, p=0.037)$, and had more comorbidities, including smoking (74.1\% vs. $35.2 \%$, p < 0.001$)$, anxiety (30.4\% vs. $23.8 \%, \mathrm{p}=0.001)$, depression $(23.0 \%$ vs. $16.2 \%, \mathrm{p}<0.001)$, and OSA $(7.5 \%$ vs. $4.9 \%, \mathrm{p}=$ 0.012). There was no significant difference between the two groups when comparing conventional cardiovascular risk factors, including hypertension (48.4\% vs. $49.1 \%, \mathrm{p}=$ $0.768)$, DM (20.9\% vs. $19.6 \%, \mathrm{p}=0.479)$, hyperlipidemia (48.4\% vs. $48.8 \%, p=0.87)$, obesity $(10.8 \%$ vs. $13.2 \%$, $\mathrm{p}=0.112), \operatorname{CKD}(9.2 \%$ vs. $8.6 \%, \mathrm{p}=0.669)$.

After propensity score matching, all baseline characteristics, including patient and hospital-level, were comparable in COPD and non-COPD groups $(p>0.05$ in all categories). All standardized mean differences between two matched groups were less than 0.1 after propensity score matching (Figure 2). For simplicity, we only showed the standardized mean difference of continuous covariate age and binary covariates in Figure 2. The dotted vertical line at 0.1 indicates the threshold below which the balance is considered to be achieved. The baseline characteristics before and after propensity score matching are shown in Table 1. 


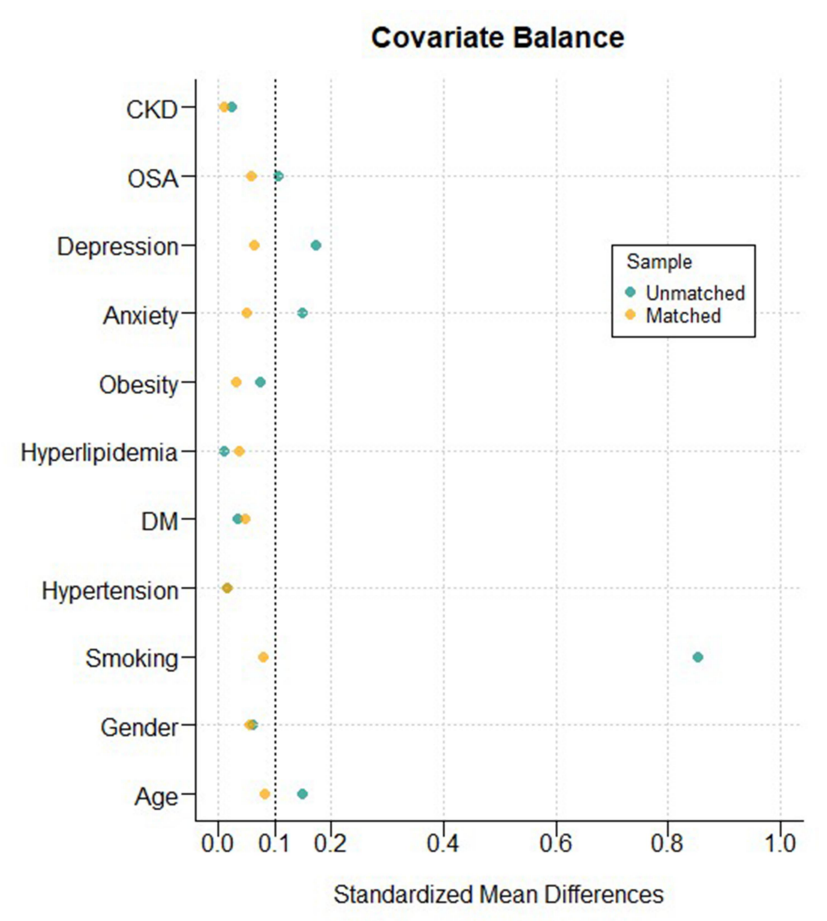

Figure 2 Standardized mean differences of covariates before and after propensity score matching between TCM patients with COPD and TCM patients without COPD. Notes: Standardized mean difference is used to examine the balance of the covariate distribution between the matched COPD group and the non-COPD group. All standardized mean differences of covariate distributions in this study were less than 0.1 , which was considered balanced.

Abbreviations: TCM, takotsubo cardiomyopathy; COPD, chronic obstructive pulmonary disease; DM, diabetes mellitus; OSA, obstructive sleep apnea; CKD, chronic kidney disease.

\section{In-Hospital Mortality}

In both the unmatched and matched cohorts, the COPD group had higher inpatient mortality compared to the nonCOPD group (unmatched $2.9 \%$ vs. $1.3 \%, p=0.006$; matched $2.9 \%$ vs. $1.0 \%$, Odds Ratio [OR] 2.93, 95\% CI 2.93, 1.39-6.15, $\mathrm{p}=0.005$ ) (Table 2).

\section{In-Hospital Complications, LOS and Total Charges}

In both the unmatched and matched cohorts, the COPD group had an increased risk of ARF compared to the nonCOPD group (unmatched, $22.5 \%$ vs. $7.7 \%, \mathrm{p}<0.001$ matched, $22.6 \%$ vs. $8.2 \%$, OR $3.25,95 \%$ CI $2.45-4.32$, $\mathrm{p}<0.001)$. After propensity score matching, the COPD group had a higher incidence of cardiogenic shock $(5.6 \%$ vs. $3.3 \%$, OR $1.76,95 \%$ CI $1.1-2.81, p=0.024)$. There was no significant difference in the two groups for incidence of cardiac arrest (unmatched, $1.6 \%$ vs. $1.8 \%, \mathrm{p}=$ 0.818 ; matched, $1.6 \%$ vs. $1.4 \%$, OR $1.16,95 \%$ CI $0.53-$ $2.54, \mathrm{p}=0.866$ ), ventricular arrhythmia (unmatched, $3.5 \%$ vs. $4.0 \%, \mathrm{p}=0.608$; matched $3.5 \%$ vs. $4.0 \%$, OR 0.88 , 95\% CI $0.53-1.46, \mathrm{p}=0.704$ ), and AKI (unmatched, $11.1 \%$ vs. $8.6 \%, \mathrm{p}=0.052$; matched, $11.2 \%$ vs. $9.3 \%$, OR $1.24,95 \%$ CI $0.9-1.7, p=0.213$ ), before or after matching. In addition, the COPD group had a longer LOS (unmatched $4.02 \pm 2.99$ vs. $3.27 \pm 3.39$ days, $\mathrm{p}<$ 0.001 ; matched $4.02 \pm 3.00$ vs. $3.40 \pm 3.54$ days, $p<$ 0.001 ) and higher total charges (unmatched 55,242.68 \pm $47,637.40$ vs. $48,316.97 \pm 47,939.84, p=0.001$; matched $55,409.23 \pm 47,809.13$ vs. $46,469.60 \pm 42,209.10, \mathrm{p}<$ 0.001 , respectively). The results can be seen in Table 2 .

\section{Discussion}

To our knowledge, this is the first study to investigate the association of COPD and in-hospital outcomes among TCM patients. COPD patients who are admitted for TCM have a higher inpatient mortality rate, higher incidence of ARF, longer LOS and higher charges, compared to those without COPD. After the propensity score matching method, COPD patients still demonstrated worse prognosis in the matched cohorts.

The prevalence of COPD was $21.8 \%$ among patients with TCM, which is slightly higher than reported in previous studies $(10.1 \%$ to $18.7 \%) .{ }^{17-19}$ One of the most wellaccepted explanations in the etiology of TCM is excessive catecholamine release related myocardial stunning. ${ }^{28}$ Previous studies found that COPD patients had a higher catecholamine level, possibly due to the over activation of the sympathetic nervous system (SNS) and the use of $\beta$ agonists in the treatment of COPD can also mimic the actions of sympathetic adrenergic stimulation. ${ }^{21,22,29,30}$ Also, many clinical studies reported the acute exacerbation of COPD as the potential triggering factor for $\mathrm{TCM}^{21-23}$ TCM and COPD may share some similar underlying pathophysiology.

In our study, TCM patients with COPD had a higher rate of in-hospital mortality and ARF than those without COPD. Several factors may have contributed to these results. First, it has been suggested that the activation of SNS and elevation of catecholamine, which are also seen in COPD patients, are associated with worse outcomes of $\mathrm{TCM}^{31}$ Second, inflammation may play a vital role in worse outcomes for TCM patients with COPD. Studies have shown that pro-inflammatory cytokine interleukin 6 (IL-6) was significantly elevated in TCM patients which may reflect endothelial and vascular smooth muscle dysfunction. In a prospective cohort study, researchers found that the increased serum levels IL- 6 of were associated 
Table I Baseline Characteristics

\begin{tabular}{|c|c|c|c|c|c|c|}
\hline \multirow[t]{2}{*}{ Variables } & \multicolumn{2}{|c|}{ Unmatched Cohort } & \multirow[t]{2}{*}{$P$ value } & \multicolumn{2}{|c|}{ Propensity-Matched Cohort } & \multirow[t]{2}{*}{$P$ value } \\
\hline & $\begin{array}{l}\text { TCM without } \\
\text { COPD }\end{array}$ & $\begin{array}{l}\text { TCM with } \\
\text { COPD }\end{array}$ & & $\begin{array}{l}\text { TCM without } \\
\text { COPD }\end{array}$ & $\begin{array}{l}\text { TCM with } \\
\text { COPD }\end{array}$ & \\
\hline $\begin{array}{l}\text { n } \\
\text { Age, (mean (sd)) }\end{array}$ & $\begin{array}{l}2455 \\
66.28(13.21)\end{array}$ & $\begin{array}{l}684 \\
68.07(10.47)\end{array}$ & 0.001 & $\begin{array}{l}1070 \\
67.09(12.74)\end{array}$ & $\begin{array}{l}678 \\
68.04(10.50)\end{array}$ & 0.107 \\
\hline $\begin{array}{l}\text { Sex, n (\%) } \\
\text { Male } \\
\text { Female } \\
\text { Unknown }\end{array}$ & $\begin{array}{l}225(9.2) \\
2229(90.8) \\
I(0.0)\end{array}$ & $\begin{array}{l}73(10.7) \\
610(89.2) \\
1(0.1)\end{array}$ & 0.306 & $\begin{array}{l}114(10.7) \\
956(89.3) \\
0(0.0)\end{array}$ & $\begin{array}{l}71(10.5) \\
606(89.4) \\
I(0.1)\end{array}$ & $0.45 I$ \\
\hline $\begin{array}{l}\text { Race, n (\%) } \\
\text { White } \\
\text { Black } \\
\text { Hispanic } \\
\text { Asian/ Pacific Islander } \\
\text { Native American } \\
\text { Other } \\
\text { Unknown }\end{array}$ & $\begin{array}{l}193 \mid(78.7) \\
156(6.4) \\
146(5.9) \\
40(1.6) \\
16(0.7) \\
60(2.4) \\
106(4.3)\end{array}$ & $\begin{array}{l}563(82.3) \\
50(7.3) \\
30(4.4) \\
5(0.7) \\
2(0.3) \\
7(1.0) \\
27(3.9)\end{array}$ & 0.037 & $\begin{array}{l}882(82.4) \\
78(7.3) \\
54(5.0) \\
5(0.5) \\
3(0.3) \\
10(0.9) \\
38(3.6)\end{array}$ & $\begin{array}{l}558(82.3) \\
50(7.4) \\
30(4.4) \\
5(0.7) \\
2(0.3) \\
7(1.0) \\
26(3.8)\end{array}$ & 0.985 \\
\hline $\begin{array}{l}\text { Patient location, } \mathbf{n}(\%) \\
\text { "Central" counties of metro areas of }\end{array}$ & $572(23.3)$ & $149(21.8)$ & 0.463 & $236(22.1)$ & $149(22.0)$ & 0.894 \\
\hline $\begin{array}{l}\text { "Finge" counties of metro areas of } \\
\geq \text { I million population }\end{array}$ & $604(24.6)$ & $160(23.4)$ & & $263(24.6)$ & $160(23.6)$ & \\
\hline $\begin{array}{l}\text { Counties in metro areas of } \\
250,000-999,999 \text { population }\end{array}$ & $608(24.8)$ & $160(23.4)$ & & $259(24.2)$ & $157(23.2)$ & \\
\hline $\begin{array}{l}\text { Counties in metro areas of } 50,000-249,999 \\
\text { population }\end{array}$ & $250(10.2)$ & $76(I I . I)$ & & III (I0.4) & 75 (II.I) & \\
\hline Micropolitan counties & $237(9.7)$ & $84(12.3)$ & & $110(10.3)$ & $82(12.1)$ & \\
\hline Non metropolitan or micropolitan counties & $176(7.2)$ & $52(7.6)$ & & $88(8.2)$ & $52(7.7)$ & \\
\hline NA & $8(0.3)$ & $3(0.4)$ & & $3(0.3)$ & $3(0.4)$ & \\
\hline $\begin{array}{l}\text { Mean household income, } \mathbf{n}(\%) \\
\$ 1-\$ 42,999 \\
\$ 43,000-\$ 53,999 \\
\$ 54,000-\$ 70,999 \\
\$ 71,000 \text { or more } \\
\text { Unknown }\end{array}$ & $\begin{array}{l}543(22.1) \\
618(25.2) \\
666(27.1) \\
594(24.2) \\
34(1.4)\end{array}$ & $\begin{array}{l}206(30.1) \\
195(28.5) \\
172(25.1) \\
107(15.6) \\
4(0.6)\end{array}$ & $<0.001$ & $\begin{array}{l}283(26.4) \\
290(27.1) \\
291(27.2) \\
202(18.9) \\
4(0.4)\end{array}$ & $\begin{array}{l}201(29.6) \\
194(28.6) \\
172(25.4) \\
107(15.8) \\
4(0.6)\end{array}$ & 0.28 \\
\hline $\begin{array}{l}\text { Primary payer, n (\%) } \\
\text { Medicare } \\
\text { Medicaid } \\
\text { Private including HMO } \\
\text { Self-pay } \\
\text { No charge } \\
\text { Other } \\
\text { Unknown }\end{array}$ & $\begin{array}{l}1404(57.2) \\
206(8.4) \\
710(28.9) \\
78(3.2) \\
4(0.2) \\
52(2.1) \\
1(0.0)\end{array}$ & $\begin{array}{l}463(67.7) \\
68(9.9) \\
118(17.3) \\
23(3.4) \\
2(0.3) \\
10(1.5) \\
0(0.0)\end{array}$ & $<0.001$ & $\begin{array}{l}677(63.3) \\
114(10.7) \\
210(19.6) \\
43(4.0) \\
2(0.2) \\
24(2.2) \\
0(0.0)\end{array}$ & $\begin{array}{l}458(67.6) \\
67(9.9) \\
118(17.4) \\
23(3.4) \\
2(0.3) \\
10(1.5) \\
0(0.0)\end{array}$ & 0.493 \\
\hline $\begin{array}{l}\text { Hospital type, n (\%) } \\
\text { Rural } \\
\text { Urban non-teaching } \\
\text { Urban teaching }\end{array}$ & $\begin{array}{l}125(5.1) \\
575(23.4) \\
1755(7 \mid .5)\end{array}$ & $\begin{array}{l}49(7.2) \\
149(2 \mid .8) \\
486(7 \mid .1)\end{array}$ & 0.092 & $\begin{array}{l}54(5.0) \\
238(22.2) \\
778(72.7)\end{array}$ & $\begin{array}{l}49(7.2) \\
149(22.0) \\
480(70.8)\end{array}$ & 0.168 \\
\hline
\end{tabular}


Table I (Continued).

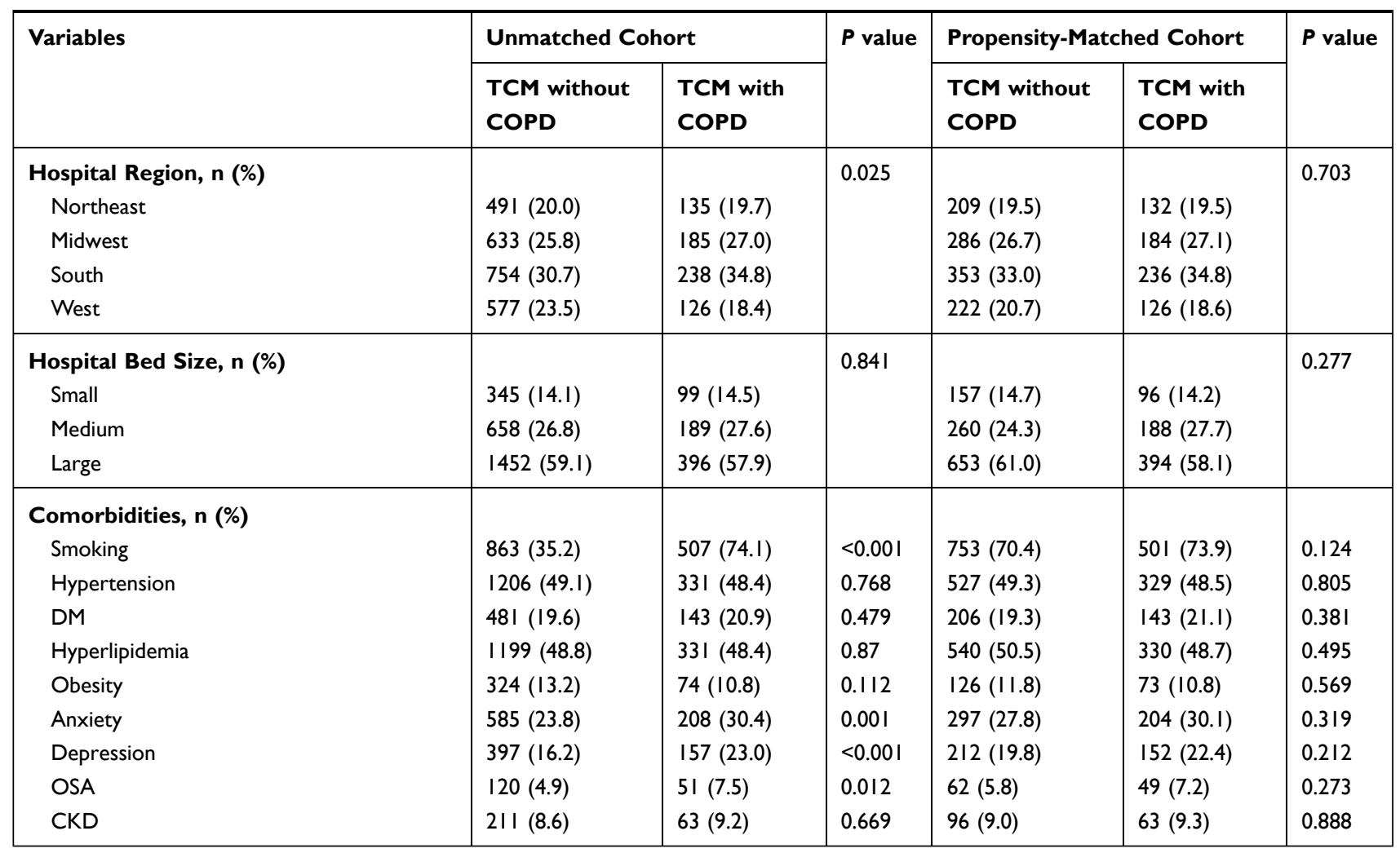

Notes: Descriptive data are shown in percentages or mean \pm standard deviation (SD), and tested with the chi-square and $t$-test for categorical and continuous variables, respectively.

Abbreviations: TCM, takotsubo cardiomyopathy; COPD, chronic obstructive pulmonary disease; DM, diabetes mellitus; OSA, obstructive sleep apnea; CKD, chronic kidney disease.

with higher adverse event rates at a mean follow-up of 178 days. ${ }^{32}$ This association remained significant even after correction for age, LVEF, and NT-proBNP levels in multivariable Cox analysis. ${ }^{33} \mathrm{COPD}$, as a chronic systemic inflammatory disease, can lead to a higher level of IL-6 than healthy individuals, ${ }^{34}$ which may contribute to the exacerbated inflammatory process and worsen outcomes. TCM and COPD combined might lead to a higher level of inflammation than patients with TCM alone.

Moreover, a study showed that TCM patients with COPD tend to present with atypical symptoms such as progressive or relapsing dyspnea, rather than typical chest pain, known as the "bronchogenic stress cardiomyopathy". ${ }^{35}$ The atypical symptoms may cause difficulty in diagnosing TCM and delay the treatment for TCM. Besides, dyspnea itself at presentation has been reported as an independent predictor of in-hospital complications, such as cardiogenic shock, ventricular arrhythmias and all-cause inpatient death, in TCM patients. ${ }^{13}$

Excessive catecholamine simulation has been thought to be one of the mechanisms of TCM and has been associated with worse outcomes in TCM patients. Though previous clinical studies yielded inconsistent results regarding the use of $\beta$-blockers in TCM patients, Kyuma M reported that intravenous propranolol can relieve the discordant ventricle by antagonizing the activation of sympathetic nerves in patients with significant intraventricular pressure gradient. ${ }^{36}$ One meta-analysis has illustrated that early using $\beta$-blockers in general TCM patients had no significant reduction in inpatient mortality. ${ }^{37}$ This discordance may be due to the heterogeneity within TCM patients. TCM patients with COPD, as a special subgroup, have a higher level of SNS activation and catecholamine stimulation, thus may benefit from $\beta$-blocker treatment. TCM patients with COPD, especially in the acute exacerbation state, are less likely to receive $\beta$ adrenergic-blockers due to the concern for bronchospasm as a side effect. This may contribute to the poor outcomes of this group of patients, though this hypothesis needs to be validated in future controlled clinical trials in subgroup patients.

Our study had several major strengths. First, we used the latest national representative database and illustrated 
Table 2 In-Hospital Outcomes and Complications

\begin{tabular}{|c|c|c|c|c|c|c|}
\hline \multirow[t]{2}{*}{ Variables } & \multicolumn{3}{|l|}{ Unmatched Cohort } & \multicolumn{2}{|c|}{ Propensity-Matched Cohort } & \multirow[t]{2}{*}{$P$ value } \\
\hline & TCM without COPD & TCM with COPD & $P$ value & TCM without COPD & TCM with COPD & \\
\hline $\mathbf{n}$ & 2455 & 684 & & 1070 & 678 & \\
\hline \multicolumn{7}{|l|}{ Outcomes } \\
\hline Death, n (\%) & $32(1.3)$ & $20(2.9)$ & 0.006 & $11(1.0)$ & $20(2.9)$ & 0.005 \\
\hline LOS, (mean (sd)) & $3.27(3.39)$ & $4.02(2.99)$ & $<0.001$ & $3.40(3.54)$ & $4.02(3.00)$ & $<0.001$ \\
\hline Total charge (mean (sd)) & $48,316.97(47,939.84)$ & $55,242.68(47,637.40)$ & 0.001 & $46,469.60(42,209.10)$ & $55,409.23(47,809.13)$ & $<0.001$ \\
\hline \multicolumn{7}{|l|}{ Complications } \\
\hline Cardiac arrest, n (\%) & $45(1.8)$ & II (I.6) & 0.818 & $15(1.4)$ & II (1.6) & 0.866 \\
\hline Cardiogenic shock, n (\%) & $106(4.3)$ & $39(5.7)$ & 0.155 & $35(3.3)$ & $38(5.6)$ & 0.024 \\
\hline Ventricular arrhythmia & $99(4.0)$ & $24(3.5)$ & 0.608 & $43(4.0)$ & $24(3.5)$ & 0.704 \\
\hline $\mathrm{AKI}, \mathrm{n}(\%)$ & $211(8.6)$ & $76(11.1)$ & 0.052 & $99(9.3)$ & $76(11.2)$ & 0.213 \\
\hline ARF, n (\%) & $188(7.7)$ & $154(22.5)$ & $<0.001$ & $88(8.2)$ & $153(22.6)$ & $<0.001$ \\
\hline
\end{tabular}

Notes: Descriptive data are shown in percentages or mean \pm standard deviation (SD), and tested with the chi-square and $t$-test for categorical and continuous variables, respectively.

Abbreviations: TCM, takotsubo cardiomyopathy; COPD, chronic obstructive pulmonary disease; AKI, acute kidney injury; ARF, acute respiratory failure; LOS, length of stay.

the association between COPD and worse inpatient mortality of TCM using propensity score matching method in an effort to reduce bias from patient and hospital-level factors, which in turn could affect the outcomes of TCM. Second, as an uncommon disease, TCM in the setting of COPD has not been well studied. The common treatment options of TCM are supportive therapy and conventional cardiovascular drugs. Our findings may offer a new view on the treatment of TCM. Lastly, the poorer outcomes in TCM patients with COPD indicate that it may be beneficial to early intervene and treat COPD, and control the activity of SNS and inflammation.

Our study had several limitations. First, as a retrospective observational design, our study can help establish associations, but not causality. Further study is warranted to verify these findings. Second, due to the nature of the NIS database, data on treatment, laboratory variables, and clinical examination results are lacking, such as the use of $\beta$-adrenergic drugs, serum catecholamine concentration, the degree of cardiac injury, and total comorbidity burden, which prevents us from analyzing those factors. Third, after the propensity score-matched analysis, we got a reduced sample size, which may affect the study's final conclusions. Fourth, we are unable to validate the ICD-10 codes used for the variables and the inpatient complications in NIS database, which may affect the accuracy of our results. Finally, the severity of COPD in this study is not specified, and therefore differences between the stable COPD and acute exacerbation period were not able to be differentiated and studied.

\section{Conclusion}

Compared with patients without COPD who are admitted to hospital for TCM, those with COPD have a higher inpatient mortality rate, a higher incidence of ARF, cardiogenic shock, longer length of stay, and higher charges. Given the available evidence in our study, prospective studies are warranted to further evaluate the impact of early intervention or treatment for COPD in short- and long-term outcomes of TCM.

\section{Abbreviations}

TCM, takotsubo cardiomyopathy; COPD, chronic obstructive pulmonary disease; AMI, acute myocardial infarction; DM, diabetes mellitus; CKD, chronic kidney disease; NIS, National Inpatient Sample; AHRQ, the Agency for Healthcare Research and Quality; LOS, length of stay; IRB, Institutional Review Board; ICD-10-CM code, the International Classification of Diseases, Tenth Revision, Clinical Modification code; OSA, obstructive sleep apnea; AKI, acute kidney injury; ARF, acute respiratory failure; SD, standard deviation; SNS, the sympathetic nervous system; IL-6, interleukin 6.

\section{Disclosure}

The authors report no conflicts of interest in this work.

\section{References}

1. Hurst RT, Prasad A, Askew JW, Sengupta PP, Tajik AJ. Takotsubo cardiomyopathy: a unique cardiomyopathy with variable ventricular morphology. JACC Cardiovasc Imaging. 2010;3(6):641-649. doi:10.1016/j.jcmg.2010.01.009 
2. Ono R, Falcão LM. Takotsubo cardiomyopathy systematic review: pathophysiologic process, clinical presentation and diagnostic approach to takotsubo cardiomyopathy. Int $J$ Cardiol. 2016;209:196-205. doi:10.1016/j.ijcard.2016.02.012

3. Templin C, Ghadri JR, Diekmann J, et al. Clinical features and outcomes of takotsubo (stress) cardiomyopathy. $N$ Engl J Med. 2015;373(10):929-938. doi:10.1056/NEJMoa1406761

4. Bietry R, Reyentovich A, Katz SD. Clinical management of takotsubo cardiomyopathy. Heart Fail Clin. 2013;9(2). doi:10.1016/j. hfc.2012.12.003

5. Prasad A, Lerman A, Rihal CS. Apical ballooning syndrome (tako-tsubo or stress cardiomyopathy): a mimic of acute myocardial infarction. Am Heart J. 2008;155(3):408-417. doi:10.1016/j. ahj.2007.11.008

6. Murugiah K, Wang Y, Desai NR, et al. Trends in short- and long-term outcomes for takotsubo cardiomyopathy among medicare fee-forservice beneficiaries, 2007 to 2012. JACC Heart Fail. 2016;4 (3):197-205. doi:10.1016/j.jchf.2015.09.013

7. Pelliccia F, Pasceri V, Patti G, et al. Long-term prognosis and outcome predictors in takotsubo syndrome: a systematic review and meta-regression study. JACC Heart Fail. 2019;7(2):143-154. doi:10.1016/j.jchf.2018.10.009

8. Agdamag AC, Patel H, Chandra S, et al. Sex differences in takotsubo syndrome: a narrative review. J Womens Health (Larchmt). 2020;29 (8):1122-1130.

9. Zaghlol R, Dey AK, Desale S, Barac A. Racial differences in takotsubo cardiomyopathy outcomes in a large nationwide sample. ESC Heart Fail. 2016;7(3):1056-1063. doi:10.1002/ehf2.12664

10. Zalewska-Adamiec M, Malyszko J, Bachórzewska-Gajewska H, Tomaszuk-Kazberuk A, Dobrzycki SJ. Takotsubo syndrome - fatal prognosis of patients with low body mass index in 5-year follow-up. Arch Med Sci. 2020;16(2):282-288.

11. Stiermaier T, Santoro F, El-Battrawy I, et al. Prevalence and prognostic impact of diabetes in takotsubo syndrome: insights from the international, multicenter GEIST registry. Diabetes Care. 2018;41 (5):1084-1088. doi:10.2337/dc17-2609

12. Kim H, Senecal C, Lewis B, et al. Natural history and predictors of mortality of patients with takotsubo syndrome. Int $J$ Cardiol. 2018;267:22-27. doi:10.1016/j.ijcard.2018.04.139

13. Bento D, Azevedo O, Santos R, et al. Short- and medium-term prognosis of takotsubo syndrome in a Portuguese population. Rev Port Cardiol. 2019;38(5):349-357. doi:10.1016/j.repc.2018.07.010

14. Yassin AS, Adegbala O, Subahi A, et al. Clinical impact of advanced chronic kidney disease on outcomes and in-hospital complications of takotsubo syndrome (broken-heart-syndrome): propensity-matched national study. Int $J$ Cardiol. 2019;277:16-19. doi:10.1016/j. ijcard.2018.09.098

15. Pauwels RA, Rabe KF. Burden and clinical features of chronic obstructive pulmonary disease (COPD). Lancet. 2004;364 (9434):613-620. doi:10.1016/S0140-6736(04)16855-4

16. Halbert RJ, Isonaka S, George D, Iqbal A. Interpreting COPD prevalence estimates: what is the true burden of disease? Chest. 2003;123(5):1684-1692. doi:10.1378/chest.123.5.1684

17. Tornvall P, Collste O, Ehrenborg E, Järnbert-Petterson H, CaseControl A. Study of risk markers and mortality in takotsubo stress cardiomyopathy. J Am Coll Cardiol. 2016;67(16):1931-1936.

18. Zalewska-Adamiec M, Bachorzewska-Gajewska H, TomaszukKazberuk A, et al. Takotsubo cardiomyopathy: serious early complications and two-year mortality - a 101 case study. Neth Heart J. 2016;24(9):511-519. doi:10.1007/s12471-016-0857-z

19. Hertting K, Krause K, Härle T, Boczor S, Reimers J, Kuck K-H. Transient left ventricular apical ballooning in a community hospital in Germany. Int $J$ Cardiol. 2006;112(3):282-288. doi:10.1016/j. ijcard.2005.09.006
20. El-Sayed AM, Brinjikji W, Salka S. Demographic and co-morbid predictors of stress (takotsubo) cardiomyopathy. Am J Cardiol. 2012;110(9):1368-1372. doi:10.1016/j.amjcard.2012.06.041

21. Rajwani A, Adam Z, Hall JA. Bronchogenic stress cardiomyopathy: a case series. Cardiology. 2015;130(2):106-111. doi:10.1159/000369296

22. White JM, Stewart RAH. Troponin elevation during exacerbations of chronic obstructive airways disease due to stress cardiomyopathy. Int J Cardiol. 2012;160(3):206-207. doi:10.1016/j.ijcard.2012.06.049

23. Peters S. Association between chronic obstructive pulmonary disease and tako tsubo cardiomyopathy - a case report. Int $J$ Cardiol. 2014;176(3):e101.

24. HCUP Nationwide Inpatient Sample (NIS), Healthcare Cost and Utilization Project (HCUP). Agency for healthcare research and quality. Rockville, MD. Available from: https://www.hcup-us.ahrq. gov/nisoverview.jsp. Accessed July 27, 2020.

25. Khera R, Krumholz HM. With great power comes great responsibility: big data research from the national inpatient sample. Circ Cardiovasc Qual Outcomes. 2017;10(7):e003846. doi:10.1161/ CIRCOUTCOMES.117.003846

26. Benedetto U, Head SJ, Angelini GD, Blackstone EH. Statistical primer: propensity score matching and its alternatives. Eur J Cardiothorac Surg. 2018;53(6):1112-1117. doi:10.1093/ejcts/ezy167

27. Stuart EA, Lee BK, Leacy FP. Prognostic score-based balance measures can be a useful diagnostic for propensity score methods in comparative effectiveness research. J Clin Epidemiol. 2013;66 (8Suppl). doi:10.1016/j.jclinepi.2013.01.013

28. Wittstein IS, Thiemann DR, Lima JAC, et al. Neurohumoral features of myocardial stunning due to sudden emotional stress. $N$ Engl $J$ Med. 2005;352(6):539-548. doi:10.1056/NEJMoa043046

29. Anand IS, Chandrashekhar Y, Ferrari R, et al. Pathogenesis of congestive state in chronic obstructive pulmonary disease. Studies of body water and sodium, renal function, hemodynamics, and plasma hormones during edema and after recovery. Circulation. 1992;86 (1):12-21. doi:10.1161/01.CIR.86.1.12

30. Keller R, Lohmann FW, Schüren KP. Catecholamines in chronic respiratory insufficiency. Respiration. 1971;28(3):273-279. doi:10.1159/000192843

31. Matsuura T, Ueno M, Iwanaga Y, Miyazaki S. Importance of sympathetic nervous system activity during left ventricular functional recovery and its association with in-hospital complications in takotsubo syndrome. Heart Vessels. 2019;34(8):1317-1324. doi:10.1007/ s00380-019-01359-4

32. Ciutac AM, Dawson D. The role of inflammation in stress cardiomyopathy. Trends Cardiovasc Med. 2020. doi:10.1016/j. tcm.2020.03.005

33. Santoro F, Tarantino N, Ferraretti A, et al. Serum interleukin 6 and 10 levels in takotsubo cardiomyopathy: increased admission levels may predict adverse events at follow-up. Atherosclerosis. 2016;254:28-34. doi:10.1016/j.atherosclerosis.2016.09.012

34. Yasuda N, Gotoh K, Minatoguchi S, et al. An increase of soluble fas, an inhibitor of apoptosis, associated with progression of COPD. Respir Med. 1998;92(8):993-999. doi:10.1016/S0954-6111(98)90343-2

35. Madias JE. 'Bronchogenic stress cardiomyopathy', a subset of takotsubo syndrome. Cardiology. 2015;131(3):160. doi:10.1159/000376571

36. Kyuma M, Tsuchihashi K, Shinshi Y, et al. Effect of intravenous propranolol on left ventricular apical ballooning without coronary artery stenosis (ampulla cardiomyopathy): three cases. Circ J. 2002;66(12):1181-1184. doi:10.1253/circj.66.1181

37. Isogai $T$, Matsui $H$, Tanaka H, Fushimi K, Yasunaga H. Early $\beta$ blocker use and in-hospital mortality in patients with takotsubo cardiomyopathy. Heart. 2016;102(13):1029-1035. doi:10.1136/ heartjnl-2015-308712 


\section{Publish your work in this journal}

The International Journal of COPD is an international, peer-reviewed journal of therapeutics and pharmacology focusing on concise rapid reporting of clinical studies and reviews in COPD. Special focus is given to the pathophysiological processes underlying the disease, intervention programs, patient focused education, and self management protocols. This journal is indexed on PubMed Central, MedLine and CAS. The manuscript management system is completely online and includes a very quick and fair peer-review system, which is all easy to use. Visit http://www.dovepress.com/testimonials.php to read real quotes from published authors. 